\title{
Dibenzofuran-induced mitochondrial dysfunction: Interaction with ANT carrier
}

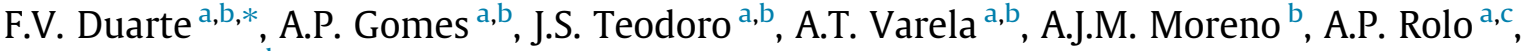 \\ C.M. Palmeira ${ }^{a, b}$ \\ ${ }^{a}$ CNC - Center for Neurosciences and Cell Biology, University of Coimbra, 3004-517 Coimbra, Portugal \\ ${ }^{\mathrm{b}}$ Department of Life Sciences, Faculty of Sciences and Technology, University of Coimbra, Apartado 3046, 3001-401 Coimbra, Portugal \\ ${ }^{\mathrm{c}}$ Department of Biology, University of Aveiro, 3810-193 Aveiro, Portugal
}

\section{A R T I C L E I N F O}

\section{Article history:}

Received 6 April 2013

Accepted 26 August 2013

Available online 3 September 2013

\section{Keywords:}

Adenine nucleotide translocator (ANT)

Cyclophilin D (CypD)

Dibenzofuran (DBF)

Mitochondria

Mitochondrial permeability transition (MPT)

\begin{abstract}
A B S T R A C T
Exposure to environmental pollutants such as dibenzofurans and furans is linked to the pathophysiology of several diseases. Dibenzofuran (DBF) is listed as a pollutant of concern due to its persistence in the environment, bioaccumulation and toxicity to humans, being associated with the development of lung diseases and cancers, due to its extremely toxic properties such as carcinogenic and teratogenic.

Mitochondria play a key role in cellular homeostasis and keeping a proper energy supply for eukaryotic cells is essential in the fulfillment of the tissues energy-demand. Therefore, interference with mitochondrial function leads to cell death and organ failure. In this work, the effects of DBF on isolated rat liver mitochondria were analyzed.

DBF exposure caused a markedly increase in the lag phase that follows depolarization induced by ADP, indicating an effect in the phosphorylative system. This was associated with a dose-dependent decrease in ATPase activity. Moreover, DBF also increased the threshold to the induction of the mitochondrial permeability transition (MPT) by calcium. Pretreatment of mitochondria with DBF also increased the concentration of carboxyatractyloside (CAT) necessary to abolish ADP phosphorylation and to induce the MPT, suggesting that DBF may interfere with mitochondria through an effect on the adenine nucleotide translocase (ANT). By co-immunoprecipitating ANT and Cyclophilin D (CypD) following MPT induction, we observed that in the presence of DBF, the ratio CypD/ANT was decreased. This demonstrates that DBF interferes with the ANT and so prevents CypD binding to the ANT, causing decreased phosphorylative capacity and inhibiting the MPT, which is also reflected by an increase in calcium retention capacity.

Clarifying the role of pollutants in some mechanisms of toxicity, such as unbalance of bioenergetics status and mitochondrial function, may help to explain the progressive and chronic evolution of diseases derived from exposure to environmental pollutants.
\end{abstract}

(c) 2013 Elsevier Ltd. All rights reserved.

\section{Introduction}

Environmental pollution has been challenging human health and healthcare practitioners more and more in the last decades. As a consequence of a wide range of industrial applications, several toxic compounds are produced, environmental release occurs and exposure becomes inevitable. Moreover, several diseases and clinical conditions have been linked to environmental pollutants exposure, such as lung inflammatory diseases (Lecureur et al., 2012), asthma (McGwin et al., 2010) and cancer (Raaschou-Nielsen et al., 2011).

Exposure to environmental contaminants such as polycyclic aromatic hydrocarbons (PAH) and halogenated aromatic hydro-

\footnotetext{
* Corresponding author at: CNC - Center for Neurosciences and Cell Biology, University of Coimbra, 3004-517 Coimbra, Portugal. Tel.: +351 239 855760; fax: +351239855789.

E-mail address: fvduarte@uc.pt (F.V. Duarte).
}

carbons (HAH), including dioxins (Yoshioka et al., 2011), plays an important role in the development of several types of human cancers. PAH and HAH are wide spread environmental contaminants that are present in cigarette smoke, coal tar and automobile exhaust. The carcinogenic effects of several PAH and $\mathrm{HAH}$ are initiated after their binding and activation of the aryl hydrocarbon receptor (AhR) (Mandal, 2005). However, there are several evidences that many compounds derived from human activity act at mitochondrial level causing its dysfunction, namely environmental pollutants and metals (MohammadiBardbori and Ghazi-Khansari, 2008; Geier et al., 2009; Tang et al., 2013).

Dibenzofuran (DBF) is an ubiquitous dioxin-like compound, but little is known about its effects, namely on primary targets such as the lung. Although some studies reported that environmental toxicants induce mitochondrial damage and impair autophagy (Palmeira and Madeira, 1997; Duarte et al., 2011, 2012), more 
systematic studies are needed in order to uncover the mechanisms underlying the toxic effects of some pollutants on mitochondria.

Mitochondria are the key organelles for energy production and many other cellular processes, including signaling pathways regulating several biologic processes in the cell. Oxidative phosphorylation (OXPHOS) in mitochondria produces more than 95\% of a cell's energy in the form of ATP under normal physiological conditions and this process involves five different protein complexes (complex $I-V$ ). Mitochondrial respiration generates most of the cellular energy (ATP) and yields several intermediate compounds involved in cellular metabolism. Therefore, perturbation of mitochondrial functions may result in severe consequences for general metabolism and all the energy transducing processes that require ATP.

Besides energy production, mitochondria participate also in several signaling pathways, regulating for example cell death/survival (Kinnally et al., 2011). An increase in mitochondrial membrane permeability is one of the key events in apoptotic and necrotic death, although the mechanisms involved are yet to be fully elucidated. It is accepted that mitochondrial permeability transition is caused by the permeability transition pore (PTP), a voltage- and $\mathrm{Ca}^{2+}$-dependent, cyclosporine $\mathrm{A}$ (CsA)-sensitive, high conductance channel, whose opening leads to permeabilization of the inner mitochondrial membrane (IMM) to solutes with molecular masses up to $1.5 \mathrm{kDa}$ (Rasola et al., 2010). Activation of mitochondrial permeability transition (MPT) is considered to be a major cause of cell death under a variety of pathophysiological conditions, including ischemia/reperfusion injury (Varela et al., 2008, 2010), neurodegenerative disease, traumatic brain injury (TBI), muscular dystrophy and drug toxicity (Millay et al., 2008; Halestrap and Pasdois, 2009; Mbye et al., 2009; Nicholls, 2009; Russmann et al., 2009).

Despite major efforts, the molecular nature of the PTP remains undefined. Earlier candidates such as the IMM adenine nucleotide translocator (ANT) and the outer mitochondrial membrane (OMM) VDAC are now considered to be regulators rather than constituents of the PTP (Rasola et al., 2010), and new putative regulators have been proposed in the recent years. Indeed, the PTP has been proposed to be regulated by proteins that interact with the OMM such as hexokinase (Pastorino and Hoek, 2008) and by ligands of the OMM translocator protein of $18 \mathrm{kDa}$, TSPO, formerly known as the peripheral benzodiazepine receptor (Sileikytè et al., 2011).

In the past, extensive analyses have dissected the factors that regulate the PTP in situ (Bernardi et al., 2006). Nonetheless, extreme caution must be exerted when these data are extrapolated to PTP modulation in the living cell. The continuous crosstalk between mitochondria and other cell compartments suggests that several proteins can play regulatory roles on the pore, connecting signaling cascades known to control the apoptotic process with the PTP, as this is a key switch in the cell commitment to death (Rasola et al., 2010).

In this work we evaluated the toxic effects of the pollutant DBF in vitro, using isolated mitochondria from rat liver. We assessed mitochondrial function (transmembrane potential generation, oxygen consumption), mitochondrial susceptibility to MPT induction and permeability of mitochondrial membrane to protons, exposing mitochondria to the pollutant.

\section{Materials and methods}

\subsection{Materials}

Dibenzofuran (DBF) was purchased from Supelco Inc. (Bellefonte, PA) (4-0261). All other reagents and chemicals used were of the highest grade of purity commercially available.

\subsection{Preparation of liver mitochondria}

All experimental procedures regarding the animals respected the guidelines of the European Directive 86/609/CEE and present in the Portuguese law. Briefly, the animals (male Wistar rats) were sacrificed by cervical dislocation and the liver was immediately removed. Mitochondria were isolated from the liver by differential centrifugation, as previously described (Rolo et al., 2009; Gomes et al., 2012). All isolation material was kept on ice during the isolation procedure. The liver was finely minced in an ice-cold homogenization buffer ( $250 \mathrm{mM}$ sucrose, $0.5 \mathrm{mM}$ ethylene glycol tetraacetic acid (EGTA), 0.5\% bovine serum albumin (BSA) and $10 \mathrm{mM}$ HEPES, pH 7.4) and the minced blood-free tissue was then homogenized with a "Potter-Elvejhem" type glass homogenizer and a Teflon piston. The homogenate was then centrifuged in a refrigerated centrifuge (Sorvall; Thermo Scientific, Rockford, IL), at $800 \mathrm{~g}$. Supernatant was collected and centrifuged at $10,000 \mathrm{~g}$ to pellet mitochondria, which were resuspended in washing medium (250 mM sucrose and $10 \mathrm{mM}$ HEPES, pH 7.4) and centrifuged again at $10,000 \mathrm{~g}$. The pellet was resuspended and finally centrifuged at $10,000 \mathrm{~g}$. Mitochondria in the pellet were resuspended in a final washing medium and immediately used. Protein content was determined by the biuret method (Gornall et al., 1949), calibrated with bovine serum albumin.

\subsection{Mitochondrial transmembrane potential $\left(\Delta \Psi_{m}\right)$ measurements}

The mitochondrial transmembrane potential $\left(\Delta \Psi_{m}\right)$ was estimated using an ion-selective electrode to measure the distribution of tetraphenylphosphonium ( $\mathrm{TPP}^{+}$) according to previously established methods (Kamo et al., 1979; Palmeira et al., 1994). The reference electrode was $\mathrm{Ag} / \mathrm{AgCl}_{2}$ (model MI 402, Microelectrodes Inc., Bedford, $\mathrm{NH}$ ). Both electrodes were connected to an adequate potentiometer (Jenway pH meter model 3305, Jenway, Essex, UK); the signal was delivered to a Kipp and Zonen recorder (model BD 121, Kipp and Zonen B.V., Delft, The Netherlands) via a manufactured potential compensatory box (Madeira, 1975). Mitochondria ( $1 \mathrm{mg}$ ) were suspended with constant stirring, at $25^{\circ} \mathrm{C}$, in $1 \mathrm{ml}$ of standard respiratory medium (130 mM sucrose, $50 \mathrm{mM} \mathrm{KCl}$, $5 \mathrm{mM} \mathrm{MgCl}_{2}, 5 \mathrm{mM} \mathrm{KH}_{2} \mathrm{PO}_{4}, 50 \mu \mathrm{M}$ EDTA, 5 mM HEPES, pH 7.4) supplemented with $3 \mu \mathrm{M} \mathrm{TPP}{ }^{+}$and $3 \mu \mathrm{M}$ rotenone, and were energized with $10 \mathrm{mM}$ succinate. A matrix volume of $1.1 \mu \mathrm{l} / \mathrm{mg}$ protein was assumed (Masini et al., 1984). Control assays were done in order to exclude the possibility of interference with the electrode.

\subsection{Mitochondrial respiration/oxygen consumption}

Oxygen consumption of isolated mitochondria was polarographically determined with a Clark oxygen electrode (Estabrook, 1967; Rolo et al., 2009) connected to a suitable recorder, through an oxygen electrode control system. Mitochondria (1 mg) were suspended under constant stirring, at $25^{\circ} \mathrm{C}$, in $1.3 \mathrm{ml}$ of standard respiratory medium described above with rotenone $(3 \mu \mathrm{M})$ as inhibitor of complex I, and energized by adding succinate to a final concentration of $10 \mathrm{mM}$. ADP (200 nmol) was added to induce state 3 respiration. The respiratory control ratio (RCR) was calculated taking into account oxygen consumption rates during state 3 (following ADP phosphorylation) and subsequent state 4 respiration. The ADP/O ratio was calculated as the nmol of ADP phosphorylated by natoms oxygen consumed (Chance and Williams, 1956).

\subsection{ATPase activity}

ATPase activity was determined spectrophotometrically at $660 \mathrm{~nm}$, in association with ATP hydrolysis (Madeira et al., 1974). The reaction was carried out at $37^{\circ} \mathrm{C}$, in $2 \mathrm{ml}$ reaction medium 
(100 mM NaCl, $25 \mathrm{mM} \mathrm{KCl,} 5 \mathrm{mM} \mathrm{MgCl}$, and $50 \mathrm{mM}$ HEPES, $\mathrm{pH}$ 7.4). After the addition of freeze-thawed mitochondria $(0.25 \mathrm{mg})$, DBF was added and allowed to incubate for $3 \mathrm{~min}$ before the initiation of reaction with the addition of $2 \mathrm{mM} \mathrm{Mg}^{2+}$-ATP, in the presence or absence of oligomycin ( $1 \mu \mathrm{g} / \mathrm{mg}$ protein). After $10 \mathrm{~min}$, the reaction was stopped by adding $1 \mathrm{ml}$ of $40 \%$ trichloroacetic acid and the samples centrifuged ( $5 \mathrm{~min}, 3000 \mathrm{~g}$ ). $2 \mathrm{ml}$ of ammonium

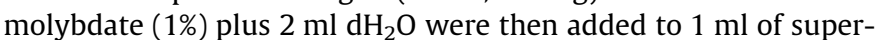
natant and reacted for $5 \mathrm{~min}$ at room temperature. ATPase activity was calculated as the difference in total absorbance and absorbance in the presence of oligomycin.

\subsection{Measurement of the mitochondrial permeability transition (MPT)}

Mitochondrial swelling associated to MPT induction was estimated by changes in light scattering as monitored spectrophotometrically at $540 \mathrm{~nm}$ (Palmeira and Wallace, 1997; Teodoro et al., 2011). The reaction medium was stirred continuously, and the temperature maintained at $25^{\circ} \mathrm{C}$. The experiments were started by the addition of $1 \mathrm{mg}$ of mitochondria to a final volume of $2 \mathrm{ml}$ of the standard incubation medium (sucrose $200 \mathrm{mM}$, $\mathrm{KH}_{2} \mathrm{PO}_{4} 1 \mathrm{mM}$, EGTA $10 \mu \mathrm{M}$ and HEPES $10 \mathrm{mM}$ ), supplemented with rotenone $3 \mu \mathrm{M}$ and succinate $5 \mathrm{mM}$. In selected assays, cyclosporine A (a known MPT inhibitor) was used as a negative control (Palmeira and Wallace, 1997). After a brief incubation period to establish a baseline absorbance, calcium was added to induce permeability transition.

\subsection{Measurement of mitochondrial calcium fluxes}

The accumulation and release of calcium by isolated mitochondria was determined using a calcium-sensitive fluorescent dye, Calcium Green-5N, as previously described (Varela et al., 2010). The reactions were carried out at $25{ }^{\circ} \mathrm{C}$ in $2 \mathrm{ml}$ of the MPT buffer (as above), supplemented with rotenone $3 \mu \mathrm{M}$ and Calcium Green-5N $200 \mathrm{nM}$. Mitochondria (1 mg) were loaded and fluorescence was recorded continuously using a Victor3 plate reader (Perkin-Elmer), with excitation and emission wavelengths of 485 and $535 \mathrm{~nm}$, respectively. Energization was obtained with $5 \mathrm{mM}$ succinate. Calcium fluxes are expressed as relative fluorescence units (RFUs).

\subsection{Co-immunoprecipitation of ANT/CypD and detection of CypD}

ANT was immunocaptured from mitochondrial extracts using monoclonal antibodies to ANT crosslinked to agarose beads (MitoSciences Cat\#MSA01). Mitochondria (1 mg) collected after MPT induction were resuspended in extraction buffer A (according to Invitrogen's Co-Immunoprecipitation kit 143.21D) and frozen in liquid nitrogen. Samples were incubated overnight at $4{ }^{\circ} \mathrm{C}$ with the antibody/agarose beads. Beads were washed four times with icecold buffer. Elution of immunocomplexes with Laemmli sample buffer and heat-denaturation were performed immediately prior to one-dimensional gel electrophoresis. Proteins were separated on 15\% SDS-polyacrylamide gels and electroblotted onto PVDF membranes. The western blots were then probed with antibodies against ANT and cyclophilin D (CypD).

\subsection{Western blotting analysis}

Western blot membranes were blocked with $5 \%$ nonfat milk and incubated with antibodies anti-ANT (MitoSciences Cat\#MSA02) or anti-CypD (Abcam ab110324) overnight at $4{ }^{\circ} \mathrm{C}$. Immunodetection was performed with WesternDot 625 (Invitrogen) goat anti-mouse western blot kits and the membranes were imaged using a Gel Doc instrument (BioRad).

\subsection{Statistic analyses}

Results are presented as means \pm SEM. of the number of different experiments (4-5 independent experiments). Solvent controls were included within each experimental determination. Statistical significance was determined using one-way ANOVA (followed by Dunnett post test), comparing all data to the control group, with the help of Prism 5 (Graphpad Software, Inc). A $P$ value $<0.05$ was considered statistically significant.

\section{Results}

\subsection{Mitochondrial membrane potential}

Transmembrane potential $\left(\Delta \Psi_{m}\right)$ sustained by mitochondria upon energization is essential for mitochondrial function; this parameter reflects the differences on electric potential and represents the greater component of the electrochemical gradient of protons in the membrane, contributing for the majority of the energy used in the oxidative phosphorylation.

We found that the $\Delta \Psi_{m}$ generated by the mitochondria isolated from rat liver was not affected by DBF exposure, comparing to control (Table 1); similarly, there were no differences in the depolarization induced when ADP was added to mitochondria (Table 1). DBF exposure also induced a dose-dependent effect on the repolarization potential of mitochondria after the phosphorylative cycle (Table 1). The lag phase preceding repolarization (period of time during which mitochondria phosphorylate all the ADP that is added) was significantly increased in mitochondria exposed to DBF, when comparing to control (Table 1), suggesting an impairment in the phosphorylative system, considering that the energization induced by substrate was not impaired.

\subsection{Mitochondrial oxygen consumption}

Oxidative phosphorylation and respiratory chain competence of mitochondria were investigated by following oxygen consumption after succinate oxidation, upon exposure to DBF. The results showed an increase in the respiratory state 2 of mitochondria exposed to DBF (Fig. 1A), which could suggest an increase in membrane permeability to protons and consequent increase in oxygen consumption in the respiratory chain. However, no differences were found when we tested the mitochondrial membrane permeability to protons assessing mitochondrial osmotic behavior in a $\mathrm{NH}_{4} \mathrm{NO}_{3}$ buffer (data not shown).

A decrease in state 3 oxygen consumption rate was observed in mitochondria exposed to DBF (Fig. 1A). Moreover, the experiments also showed that, if state 3 respiratory rate was not significantly decreased, phosphorylation lag phase was already taking longer when DBF was present. This supports the enlarged lag phase reported previously and suggests that DBF impair the mitochondrial phosphorylative system. Additionally, respiratory state 4 was clearly increased in mitochondria exposed to DBF (Fig. 1A), reflecting the impairment in mitochondrial function and a possible disruption of mitochondrial membrane impermeability after phosphorylation and consequent forced respiration. Consequently, both RCR and ADP/O ratios were decreased in mitochondria exposed to the pollutant (Fig. 1B).

\subsection{ATPase activity}

To try to elucidate the increased lag phase and impairment during phosphorylation, we assessed mitochondrial ATPase activity; the results showed that DBF caused a decrease in the ATPase activity for all the concentrations tested (Fig. 1C). The decrease in the 
Table 1

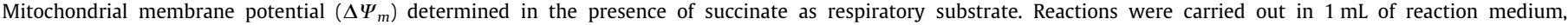

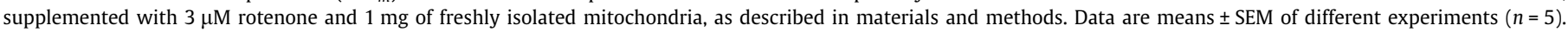
Values statistically different from control.

\begin{tabular}{|c|c|c|c|c|c|c|}
\hline & Control & $\begin{array}{l}\text { DBF } \\
10 \mu \mathrm{M}\end{array}$ & $\begin{array}{l}\text { DBF } \\
50 \mu \mathrm{M}\end{array}$ & $\begin{array}{l}\text { DBF } \\
100 \mu \mathrm{M}\end{array}$ & $\begin{array}{l}\text { DBF } \\
150 \mu \mathrm{M}\end{array}$ & $\begin{array}{l}\mathrm{DBF} \\
250 \mu \mathrm{M}\end{array}$ \\
\hline$\Delta \Psi_{m}(\%$ control $)$ & 100 & $100.2 \pm 0.25$ & $100 \pm 0.42$ & $100.4 \pm 0.46$ & $99.7 \pm 0.75$ & $99.2 \pm 0.74$ \\
\hline$\Delta \Psi_{\mathrm{ADP}}(\%$ control $)$ & 100 & $104.4 \pm 2.59$ & $104.7 \pm 5.08$ & $107.7 \pm 6.44$ & $103.2 \pm 1.84$ & $101.9 \pm 2.42$ \\
\hline Repolarization (\% control) & 100 & $100.1 \pm 0.3$ & $99.8 \pm 0.46$ & $99.4 \pm 0.51$ & $97.8 \pm 0.75^{*}$ & $95.8 \pm 1.07^{*}$ \\
\hline Lag phase (\% control) & 100 & $111.4 \pm 1.17^{*}$ & $122.4 \pm 6.43^{*}$ & $120.3 \pm 7.71^{*}$ & $145.0 \pm 8.15^{*}$ & $167.2 \pm 10.49^{*}$ \\
\hline
\end{tabular}

$P<0.05$.

A

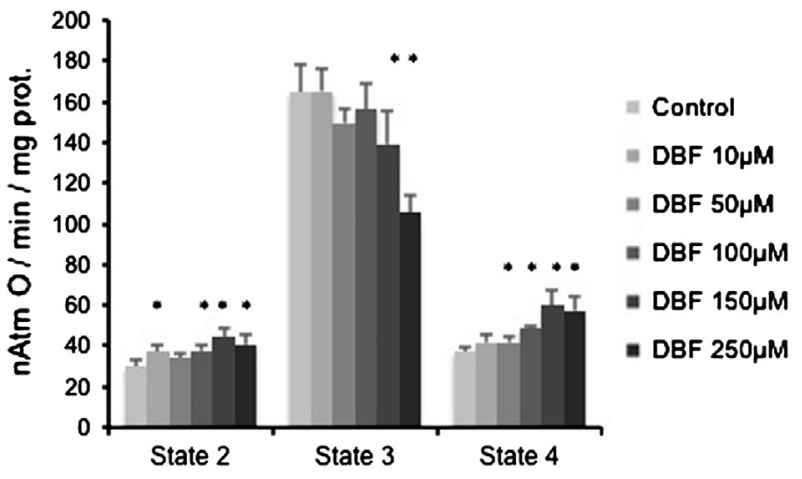

B
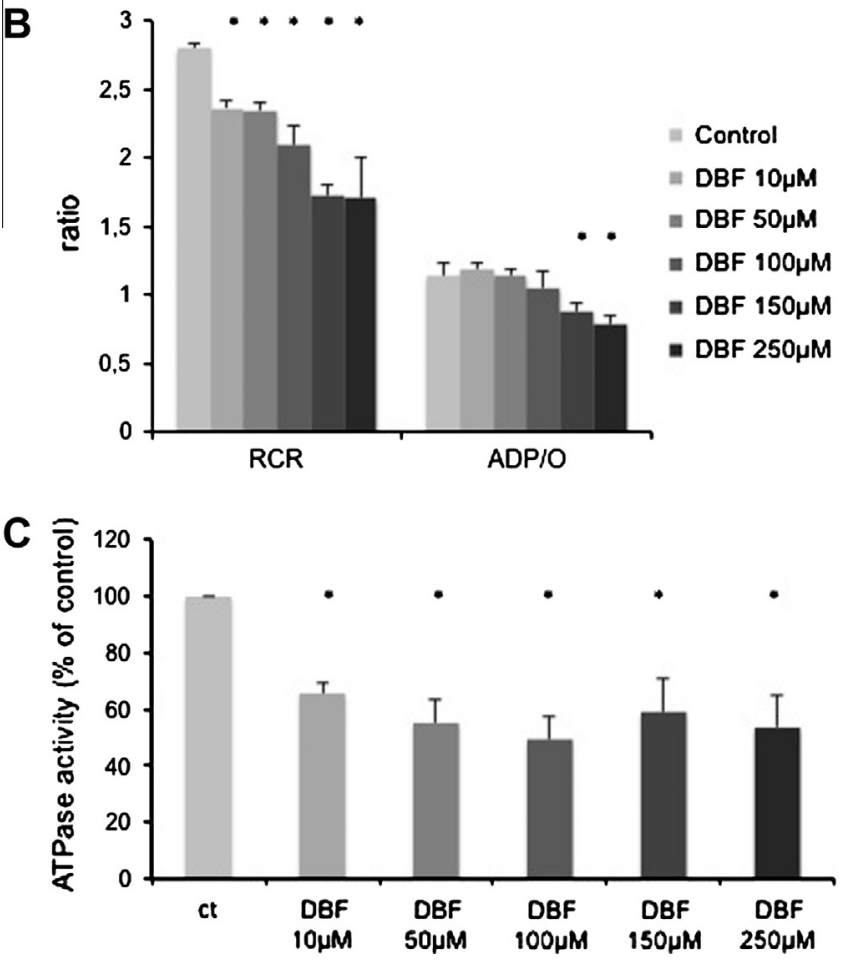

Fig. 1. Effects of Dibenzofuran on mitochondrial oxygen consumption. (A) State 2 state 3 and state 4 respiration in liver mitochondria, upon incubation with DBF. Reactions were carried out in $1.3 \mathrm{ml}$ of reaction medium, supplemented with $3 \mu \mathrm{M}$ rotenone and $1 \mathrm{mg}$ of freshly isolated mitochondria. Oxygen consumption was polarographically determined with a Clark oxygen electrode as described in Materials and Methods. Energization was achieved with $10 \mathrm{mM}$ succinate and phosphorylation induced by $200 \mathrm{nmol}$ ADP. (B) Effects of Dibenzofuran in mitochondrial RCR and ADP/O ratios. (C) Mitochondrial ATPase activity measured spectrophotometrically in mitochondria upon incubation with Dibenzofuran. Data are means \pm SEM of different experiments $(n=5)$. Values statistically different from control: ${ }^{*} P<0.05$.

ATPase activity can explain the longer lag phase but does not exclude that DBF can act on another component of the phosphorylative system (e.g. ANT - adenine nucleotide translocator).

\subsection{Mitochondrial swelling and MPT induction}

The effects of DBF on mitochondrial permeability transition (MPT) were assessed using light scattering to monitor mitochondrial swelling. We found that DBF reduced mitochondrial calcium-induced and CsA-sensitive osmotic swelling (Fig. 2A), suggesting that the pollutant can inhibit the permeability transition to some extent. This inhibition of MPT pointed out to an effect of DBF in one particular component of the phosphorylative system that is also enrolled in the permeability transition, the adenine nucleotide translocator (ANT). In order to identify the mechanism by which DBF prevents MPT induction, DBF effects were investigated in conditions of carboxyatractyloside (CAT)-induced MPT. CAT is a highly selective inhibitor of cytosolic side-specific ANT that causes stabilization of the $c$ conformation of ANT, inducing MPT.

Using CAT also in mitochondrial swelling experiments, we could see that DBF prevents mitochondrial swelling that is induced by CAT (Fig. 2B), and we also compared this prevention with that provided by bongkrekic acid (BKA), which blocks ANT in a conformation that prevents MPT. Both DBF and BKA inhibit MPT induction and consequent mitochondrial swelling (Fig. 2B), suggesting that DBF is exerting an effect on ANT.

To test the hypothesis that DBF interacts with ANT, a titration with CAT was performed. After adding succinate, mitochondria developed a potential of about $-220 \mathrm{mV}$ (negative inside). With the addition of ADP, the potential dropped because ATP synthase uses $\Delta \Psi_{m}$ to phosphorylate ADP. After a short lag phase, when ADP phosphorylation takes place, the transmembrane potential repolarized close to the initial value. Successive additions of CAT aliquots before ADP addition, as indicated in Fig. 3, progressively block a higher number of ANT units contributing to the observed decreased number of phosphorylation cycles induced by successive ADP additions, as well as to the observed increasing in lag phase corresponding to successive phosphorylation cycles.

Measuring mitochondrial membrane potential after ADP addition we found that, with DBF, less CAT was needed to completely abolish phosphorylation/repolarization (Fig. 4), indicating that mitochondria incubated with DBF already had ANT inhibition and thus an impaired phosphorylative system.

\subsection{Calcium retention capacity}

DBF effects on MPT induction were also tested assessing calcium retention capacity in mitochondria, using the fluorescent probe Calcium-Green $5 \mathrm{~N}$. These experiments showed that DBF exposure induced an increase in the calcium retention capacity after energization of mitochondria, when compared to control (Fig. 5A). DBF caused a delay in calcium release from mitochondria, that is the consequence of PTP opening and MPT induction, a CsAsensitive event (Fig. 5A). Analyzing calcium retention capacity in mitochondria we found that CAT greatly induces MPT (Fig. 5B); 

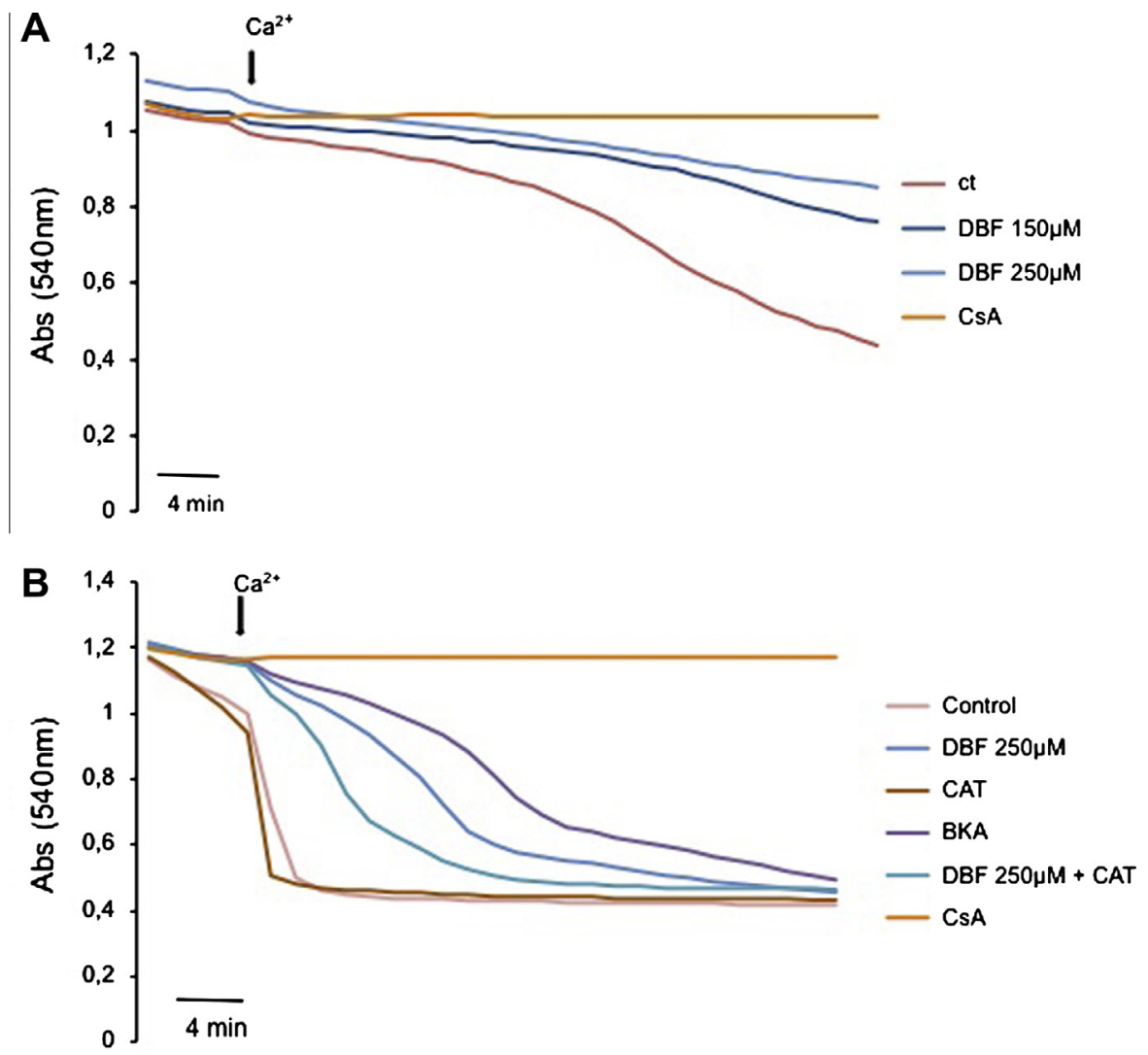

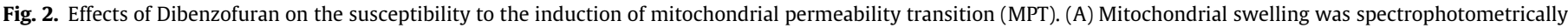

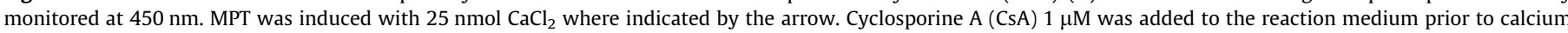

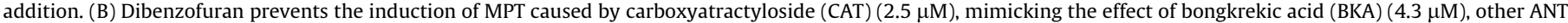

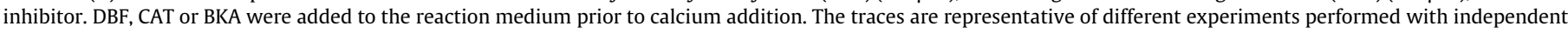
mitochondrial preparations $(n=5)$.

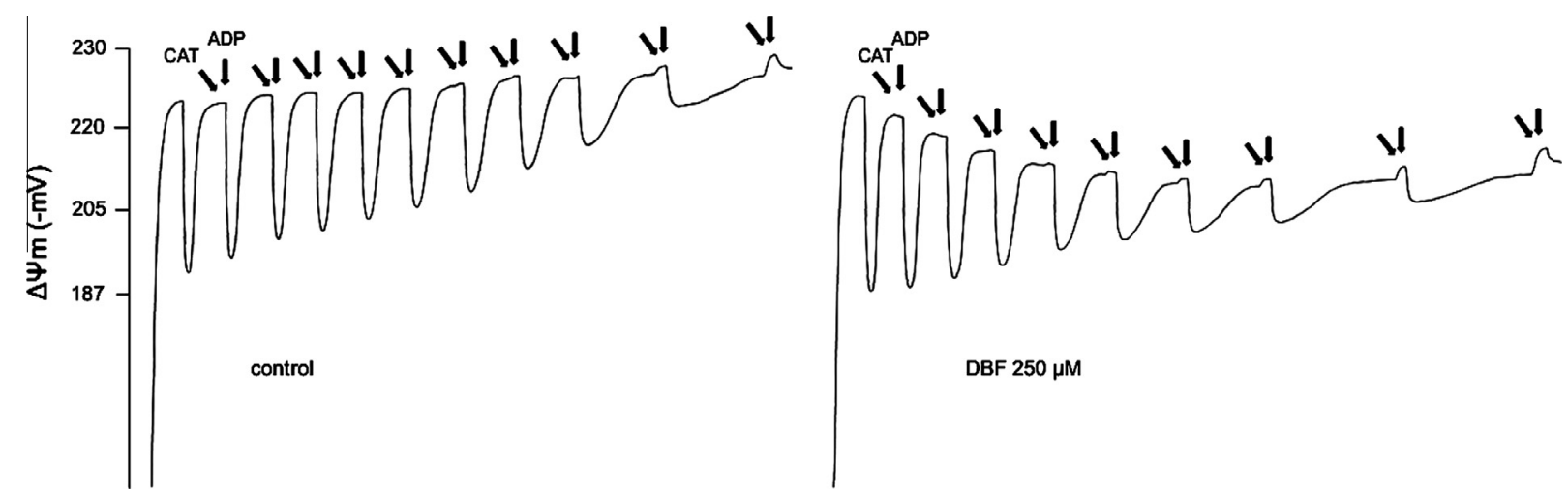

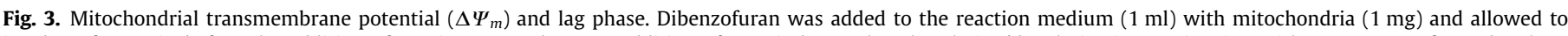

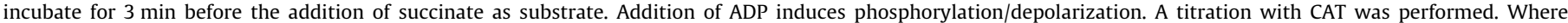
indicated, CAT and ADP aliquots were added. The traces represent typical direct recordings from independent experiments $(n=5)$.

DBF exposure prevents this from occurring, also when CAT is present, and again it displays an effect similar to BKA (Fig. 5B).

\subsection{Co-immunoprecipitation of ANT/CypD}

MPT induction is dependent on CypD binding to ANT, giving that CypD association facilitates a conformational change in the ANT, hence sensitizing to calcium and promoting MPT. Using mitochondria samples that were used in MPT induction experiments, we immunoprecipitated ANT and then used western blot to evaluate CypD present in the precipitate (thus bound previously to ANT).
When MPT induction is prevented, either with CsA or DBF exposure, the ratio CypD/ANT in the sample is decreased when compared to control or CAT (a representative image is shown in Fig. 6), also suggesting that DBF was interacting with ANT and so preventing CypD binding and MPT induction.

\subsection{PTP activation by mitochondrial outer membrane translocator protein (TSPO)}

In the last years, several other proteins have been reported to modulate PTP opening, namely the mitochondrial outer membrane 


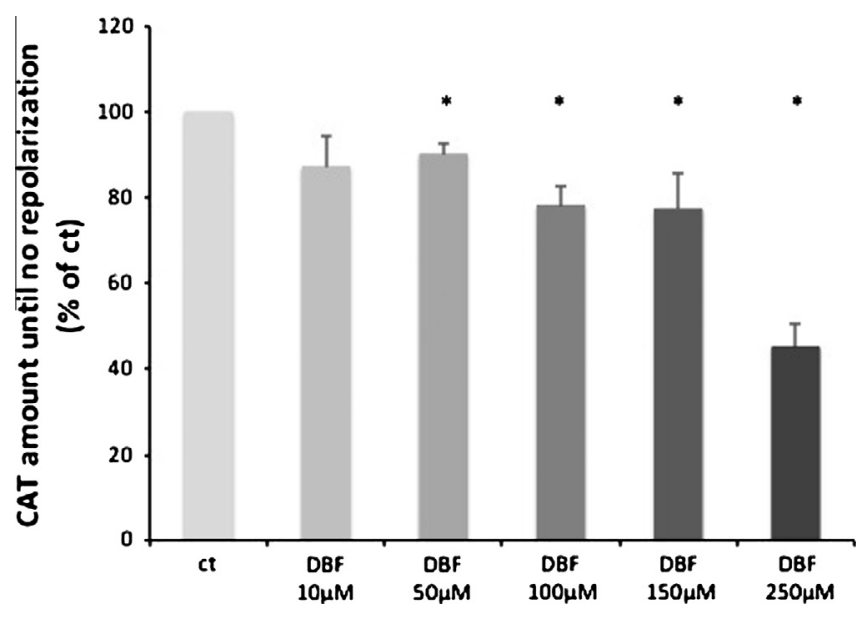

Fig. 4. Inhibition of mitochondrial ANT carrier by carboxyatractyloside (CAT). Mitochondrial transmembrane potential $\left(\Delta \Psi_{m}\right)$ was measured as previously and a titration with CAT was performed until no depolarization/repolarization was observed when ADP was added to mitochondria. Data are means \pm SEM of different experiments $(n=5)$. Values statistically different from control: ${ }^{*} P<0.05$.

translocator protein of $18 \mathrm{kDa}$, TSPO, formerly known as the peripheral benzodiazepine receptor. We wanted to check if DBF was also interacting with this receptor and this way modulating MPT induction. We performed calcium retention experiments using a ligand for TSPO, PK11195, which induces pore opening and consequent calcium release (Fig. 7). However, when DBF was present, no differences were found in calcium retention capacity of mitochondria challenged with the ligand (PK11195), suggesting that DBF effects were not being exerted via TSPO binding (Fig. 7).

\section{Discussion}

Environmental pollutants such as dioxins and furans are extremely toxic and related with several diseases, such as cancer. Some toxicology studies have addressed health effects related to exposure to chemicals, but few have described the molecular targets that cause toxicity (Yoshioka et al., 2011).

DBF is an aromatic ether with properties and chemical structure similar to dioxins, and potential exposure to DBF may occur through inhalation and dermal contact, particularly at sites engaged in combustion/carbonization processes. This work focused on the toxic effects of DBF directly on mitochondria, using an in vitro approach, given that not many studies have been conducted concerning mitochondrial harming by environmental pollutants. We have previously reported some of the effects caused by DBF, either for mitochondria isolated from lungs (Duarte et al., 2011) or lung cells in culture (Duarte et al., 2012). Given the importance of mitochondria for organisms, to evaluate toxic effects that impair mitochondrial function is necessary when one wants to study the toxicity of a given environmental pollutant.

Binding to AhR and related ROS generation has been suggested as the mechanism of action underlying some of the toxic and biological effects of these dioxins and dioxin-like compounds (Chiba et al., 2011). However, the core mechanisms are not fully understood and toxic mechanisms activated by dioxins and dioxin-like compounds, independent of AhR activation, must be considered (Ahmed et al., 2005). Recently, siRNA-mediated knockdown of the AhR in lung epithelial cells and fibroblasts was shown to increase sensitivity to smoke-induced apoptosis (Rico de Souza et al., 2011). This involved mitochondrial dysfunction, decreased antioxidant enzymes and oxidative stress.
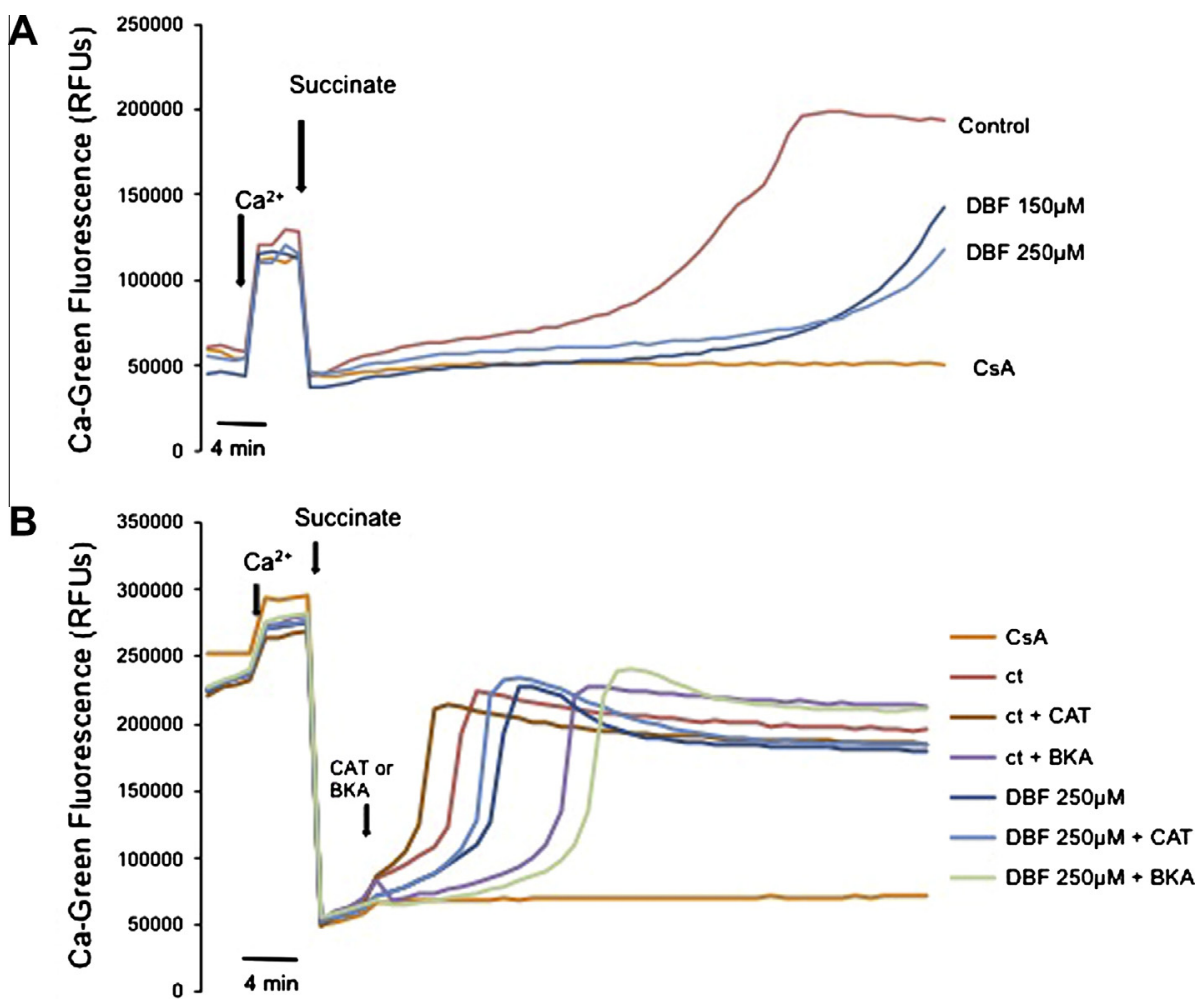

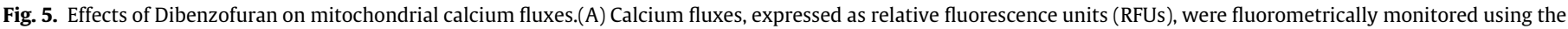

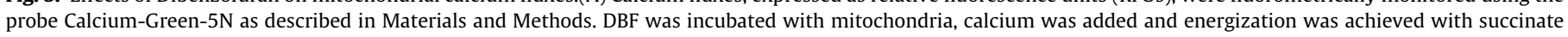

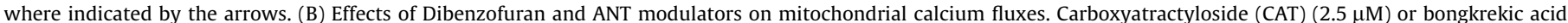

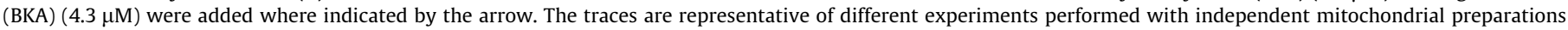
$(n=5)$. 


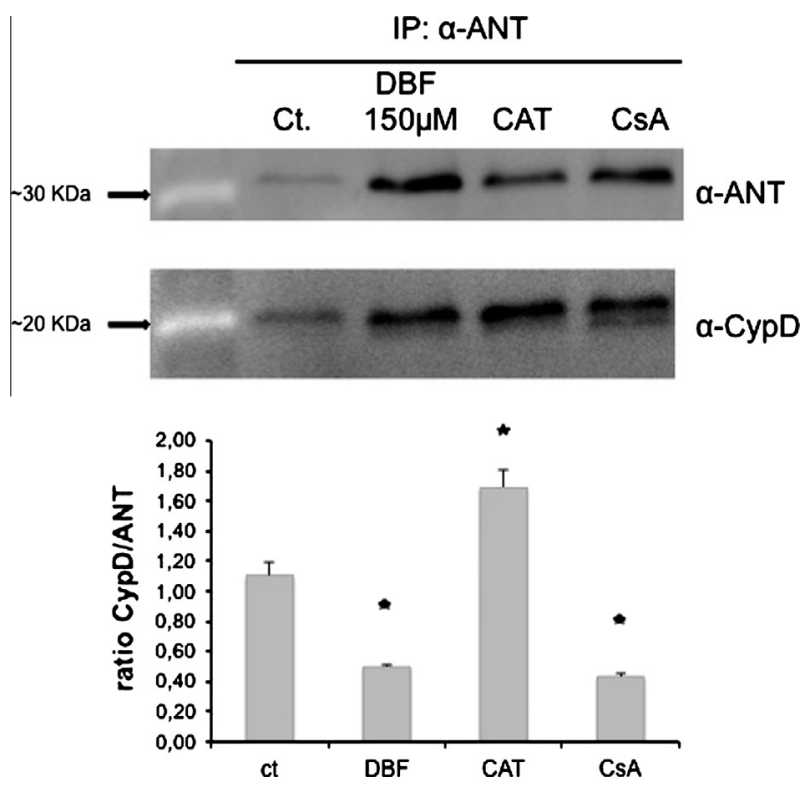

Fig. 6. Immunoprecipitation of ANT and CypD from mitochondrial extracts. Mitochondrial samples used in MPT experiments were collected, as described in Materials and Methods. Western blots were performed using antibodies against ANT and CypD proteins to evaluate its presence in the immunoprecipitates, and the arrows indicate the molecular weight markers in the first lane. To quantify the intensity of the bands obtained via Western blot analysis, we applied Image J software-based analysis (http://www.rsb.info.nih.gov/ij). The figure contains a representative immunoblot $(n=4)$. Values statistically different from control: ${ }^{*} P<0,05$.

Mitochondria are key organelles in cellular function. Their main role is to generate energy (ATP) for cellular function, but they also participate in several other processes such as calcium homeostasis, free radicals generation and apoptosis (Orrenius et al., 2007; Contreras et al., 2010). The study of drug-induced mitochondrial toxicity may elucidate distinct mechanisms by which drugs interfere with energy production by the cell.

The results indicate that mitochondria are targeted by DBF-induced toxicity. Although DBF did not affect mitochondrial capacity to generate and sustain membrane potential, a decrease in the mitochondrial phosphorylative efficiency seems to be a critical event in DBF-induced damage. Similar mitochondrial impairment had already been reported for exposure to other toxicants (Simões et al., 2010). Although $\Delta \Psi_{m}$ developed after succinate energization was not affected, the lag phase that occurs in the phosphorylative cycle and precedes repolarization was increased in mitochondria incubated with DBF when compared to control. This reflects an impairment in the phosphorylative system, which causes a delay in the restoration of membrane potential after conversion of ADP to ATP. Inversely, Jaiswal and colleagues have already reported a decrease in mitochondrial membrane potential when exposing HepG2 liver cells to DBF (Jaiswal et al., 2012); however, this unlike effects at mitochondrial level can be partially explained by the avowed fact that DBF-derived metabolites induce significantly higher cytotoxicity in HepG2 cells than its parent compound (DBF). Other authors studying environmental toxicants also reported toxic effects that include decreased oxygen consumption and decreased mitochondrial activity in the liver (Roos et al., 2011), while studies focused on the hepatotoxicity of synthetic compounds also described impairment of mitochondrial phosphorylation efficiency, decreased state 3 respiration and ATP levels, and increased lag phase, without affecting the membrane potential (Silva et al., 2013). When measuring oxygen consumption rate by mitochondria, a decrease in respiratory state 3 (active phosphorylation) was detected after exposure to DBF.

Mitochondria exposed to DBF did not recover the state 4 respiration values after phosphorylation, showing that DBF affects mitochondrial integrity and coupling of oxidative phosphorylation. Consequently, both respiratory control ratio (RCR) and efficiency of ATP synthesis coupled to mitochondrial respiration, referred to as the $\mathrm{ADP} / \mathrm{O}$ ratio, were decreased in mitochondria exposed to DBF when comparing to control. In both hepatic mitochondria isolated from TCDD-treated mice and mitochondria incubated in vitro with TCDD, a number of functional alterations have been reported, including a defect in ATP synthesis (Shertzer et al., 2006; Kopf and Walker, 2010). In primary hepatocytes, TCDD has been shown to induce mitochondrial dysfunction (Aly and Domènech, 2009). To further explore and understand the cause for the impairment in phosphorylative system, and the oxygen consumption rates, we

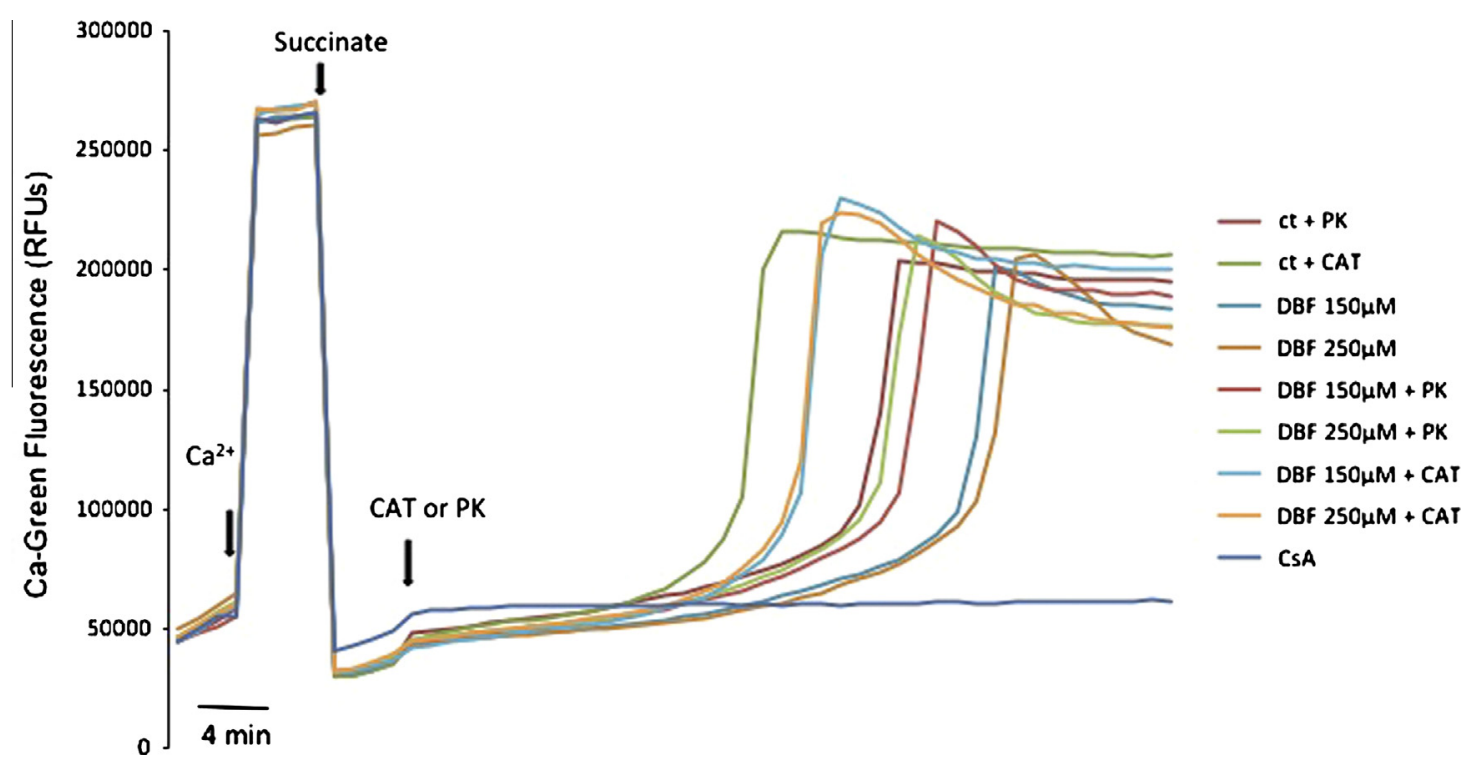

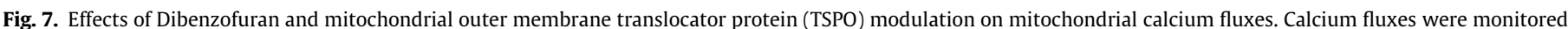

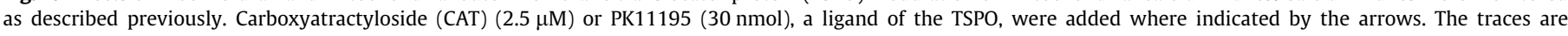
representative of different experiments performed with independent mitochondrial preparations $(n=5)$. 
evaluated ATPase activity, since other component of the phosphorylative system should be impaired, in order to justify the evident increase in mitochondrial lag phase during phosphorylation. Indeed, ATPase activity was decreased in mitochondria exposed to DBF. Although, the defects in ATPase efficiency did not fully match the "dose-dependent" increase in lag phase detected in the membrane potential experiments, and this effect does not exclude action at the level of other components of the phosphorylative system.

Silva et al. described a decrease in the phosphorylation capacity of liver mitochondria as a consequence of the interaction of a synthetic retinoid with the components of the phosphorylative system (Silva et al., 2013), namely an inhibitory effect on the ANT. The authors also reported an induction of MPT through the interaction with the ANT, arguing that those effects constitute a potential mechanism for acitretin-induced cell death by apoptosis and necrosis and toxicity in liver cells. Other authors report that survival of tumor cells is favored by mitochondrial changes that make death induction more difficult in a variety of stress conditions (such as exposure to chemotherapeutics), and that these changes include unbalance of the redox equilibrium and inhibition of MPT (Chiara et al., 2012). Having detected an inhibition of MPT elicited by DBF, we considered that a common player of both phosphorylation and MPT was a target for DBF toxicity. Experiments in order to evaluate MPT induction, a phenomenon that results from the opening of the so called mitochondrial permeability transition pore (MPTP) showed that this toxicant prevented MTP induction, when compared with control mitochondria. In a similar way, Sang and colleagues recently reported an "MPT-independent" toxicity caused by an environmental toxicant (Sang et al., 2012). Thus, although the impairment of ATP synthesis in hepatic mitochondria, data prompted us the idea that DBF was interacting with ANT, and so DBF was somehow mimicking CsA or BKA to inhibit induction of the MPT and prevent mitochondrial swelling, which can ultimately help to explain a tumorigenic phenotype, due to increased ability to escape cell death.

With the results pointing out an interaction of DBF with ANT as the cause for the decreased efficiency of ATP production and inhibition of MPT, a titration with CAT was performed to better understand DBF effects on mitochondrial transmembrane potential. CAT is a highly selective inhibitor of cytosolic side-specific mitochondrial ANT that causes stabilization of the $c$ conformation of ANT leading to MPTP opening. The results showed that, in mitochondria incubated with DBF, less CAT was needed to completely inhibit de/ repolarization after ADP addition. On the other hand, mitochondrial lag phase during a phosphorylative cycle was larger when we added CAT to mitochondria incubated with DBF, compared to control. This showed that, when exposed to DBF, mitochondria already had some ANT inhibition, supporting the DBF/ANT interaction and consequent impairment of the translocase's function. Using CAT also in mitochondrial swelling experiments, we could see that DBF prevented mitochondrial swelling that is induced by CAT, and we also compared this prevention with that provided by BKA, observing that both DBF and BKA inhibit MPT induction and consequent mitochondrial swelling. Additional experiments were performed using the fluorescent probe calcium-green to assess calcium retention capacity. We confirmed that DBF inhibited MPT induction, not only when compared only to control situation but also preventing CAT effect on the MPT triggering. Taking into account the suggested inhibition of the ANT activity, based on the lag phase experiments, DBF inhibitory effect on the MPT is probably dependent on the ANT.

In order to elucidate the involvement of DBF in the MPTP opening and the role of an interaction with ANT, we performed immunoprecipitation to check this interaction. It is known that, during mPTP opening, CypD interactions with ANT promote mPTP open- ing and MPT induction (Hansson et al., 2011). We could see that, in samples that were used in MPT induction experiments, immunoprecipitating ANT we could detect CypD (thus bound previously to ANT). When MPT induction was prevented, either with CsA or $\mathrm{DBF}$ exposure, the ratio CypD/ANT in the sample was decreased when compared to control or CAT, also supporting that DBF was interacting with ANT and so preventing CypD binding and MPT induction. Nonetheless, we also wanted to assess if DBF was interacting with other protein that has been reported to modulate MPTP opening, the mitochondrial outer membrane translocator protein of $18 \mathrm{kDa}$, TSPO, formerly known as the peripheral benzodiazepine receptor (Sileikyte et al., 2011). Some studies have shown that TSPO can be affected by oxidative stress, as well as contribute to oxidative stress at mitochondrial level and be involved in damaging effects of anthracene in the liver (Dimitrova-Shumkovska et al., 2010). Calcium retention capacity experiments were performed using a ligand for TSPO, PK11195, which induces MPT and consequent calcium release. However, when DBF was present, no differences were found in calcium retention capacity of mitochondria challenged with the ligand (PK11195), suggesting that DBF effects were not being exerted via TSPO binding and following MPT modulation. Many times, the apoptotic and survival pathways are activated in parallel in response to environmental toxicity; the balance towards either cell death or survival depends in most cases on the intensity and length of the exposure.

In summary, this work shows that DBF impairs hepatic mitochondrial function. DBF toxicity is mainly related to a decrease in phosphorylative efficiency, shown by an increase in lag phase. Also, a possible interaction of DBF with ANT carrier is supported by our results and induction of mitochondrial permeability transition is also affected by DBF exposure. Yet, further studies focused on understanding the upstream molecular mechanisms by which metabolic pathways are deregulated are needed. Those may ultimately help to develop therapeutic strategies and to prevent diseases related to pollutants exposure.

\section{Conflicts of interest}

The authors declare that there are no conflicts of interest.

\section{Acknowledgements}

This work was supported by Fundação para a Ciência e a Tecnologia, Portugal (grant SFRH/BD/38372/2007 to FVD, grant SFRH/BD/ $44674 / 2008$ to APG, grant SFRH/BD/38467/2007 to JST and grant SFRH/BD/44796/2008 to ATV).

\section{References}

Ahmed, S., Shibazaki, M., Takeuchi, T., Kikuchi, H., 2005. Protein kinase Ctheta activity is involved in the 2,3,7,8-tetrachlorodibenzo-p-dioxin-induced signal transduction pathway leading to apoptosis in L-MAT, a human lymphoblastic Tcell line. FEBS J. 272, 903-915.

Aly, H.A.A., Domènech, O., 2009. Cytotoxicity and mitochondrial dysfunction of 2,3,7,8-tetrachlorodibenzo-p-dioxin (TCDD) in isolated rat hepatocytes. Toxicol. Lett. 191, 79-87.

Bernardi, P., Krauskopf, A., Basso, E., Petronilli, V., Blachly-Dyson, E., Di Lisa, F., Forte, M.A., 2006. The mitochondrial permeability transition from in vitro artifact to disease target. FEBS J. 273 (10), 2077-2099.

Chance, B., Williams, G., 1956. The respiratory chain and oxidative phosphorylation. Adv. Enzymol. Relat. Subjects Biochem. 17, 65-134.

Chiara, F., Gambalunga, A., Sciacovelli, M., Nicolli, A., Ronconi, L., Fregona, D., Bernardi, P., Rasola, A., Trevisan, A., 2012. Chemotherapeutic induction of mitochondrial oxidative stress activates GSK-3 $\alpha / \beta$ and Bax, leading to permeability transition pore opening and tumor cell death. Cell Death and Disease, 3, e444. http://dx.doi.org/10.1038/cddis.2012.184

Chiba, T., Uchi, H., Tsuji, G., Gondo, H., Moroi, Y., Furue, M., 2011. Arylhydrocarbon receptor (AhR) activation in airway epithelial cells induces MUC5AC via reactive oxygen species (ROS) production. Pulm. Pharmacol. Ther. 24, 133-140.

Contreras, L., Drago, I., Zampese, E., Pozzan, T., 2010. Mitochondria: the calcium connection. Biochim. Biophys. Acta 1797 (6-7), 607-618. 
Dimitrova-Shumkovska, J., Veenman, L., Ristoski, T., Leschiner, S., Gavish, M., 2010. Decreases in binding capacity of the mitochondrial $18 \mathrm{kDa}$ translocator protein accompany oxidative stress and pathological signs in rat liver after DMBA exposure. Toxicol. Pathol. 38, 957-968.

Duarte, F.V., Simões, A.M., Teodoro, J.S., Rolo, A.P., Palmeira, C.M., 2011. Exposure to dibenzofuran affects lung mitochondrial function in vitro. Toxicol. Mech. Methods 21 (8), 571-576.

Duarte, F.V., Teodoro, J.S., Rolo, A.P., Palmeira, C.M., 2012. Exposure to dibenzofuran triggers autophagy in lung cells. Toxicol. Lett. 209 (1), 35-42.

Estabrook, R., 1967. Mitochondrial respiratory control and the polarographic measurement of ADP/O ratios. Methods Enzymol 10, 41-47.

Geier, D.A., King, P.G., Geier, M.R., 2009. Mitochondrial dysfunction, impaired oxidative-reduction activity, degeneration, and death in human neuronal and fetal cells induced by low-level exposure to thimerosal and other metal compounds. Toxicol. Environ. Chem., 1-15, iFirst.

Gomes, A.P., Duarte, F.V., Nunes, P., Hubbard, B.P., Teodoro, J.S., Varela, A.T., Jones, J.G., Sinclair, D.A., Palmeira, C.M., Rolo, A.P., 2012. Berberine protects against high fat diet-induced dysfunction in muscle mitochondria by inducing SIRT1dependent mitochondrial biogenesis. Biochim. Biophys. Acta 1822 (2), 185-195.

Gornall, A., Bardawill, C., David, M., 1949. Determination of serum proteins by means of the biuret reaction. J. Biol. Chem. 177 (2), 751-766.

Halestrap, A.P., Pasdois, P., 2009. The role of the mitochondrial permeability transition pore in heart disease. Biochim. Biophys. Acta 1787 (11), 1402-1415.

Hansson, M.J., Morota, S., Chen, L., Matsuyama, N., Suzuki, Y., Nakajima, S., Tanoue, T., Omi, A., Shibasaki, F., Shimazu, M., Ikeda, Y., Uchino, H., Elmér, E., 2011. Cyclophilin D-sensitive mitochondrial permeability transition in adult human brain and liver mitochondria. J. Neurotrauma 28 (1), 143-153.

Jaiswal, P.K., Srivastava, S., Gupta, J., Thakur, I.S., 2012. Dibenzofuran induces oxidative stress, disruption of trans-mitochondrial membrane potential $\left(\Delta \Psi_{m}\right)$ and G1 arrest in human hepatoma cell line. Toxicol. Lett. 214 (2), 137-144.

Kamo, N., Muratsugu, M., Hongoh, R., Kobatake, Y., 1979. Membrane potential of mitochondria measured with an electrode sensitive to tetraphenyl phosphonium and relationship between proton electrochemical potential and phosphorylation potential in steady state. J. Membr. Biol. 49 (2), 105-121.

Kinnally, K.W., Peixoto, P.M., Ryu, S.Y., Dejean, L.M., 2011. Is mPTP the gatekeeper for necrosis, apoptosis, or both? Biochim. Biophys. Acta 1813 (4), 616-622.

Kopf, P.G., Walker, M.K., 2010. 2,3,7,8-tetrachlorodibenzo-p-dioxin increases reactive oxygen species production in human endothelial cells via induction of cytochrome P4501A1. Toxicol. Appl. Pharmacol. 245, 91-99.

Lecureur, V., Arzel, M., Ameziane, S., Houlbert, N., Le Vee, M., Jouneau, S., Fardel, O. 2012. MAPK- and PKC/CREB-dependent induction of interleukin-11 by the environmental contaminant formaldehyde in human bronchial epithelial cells. Toxicology $292(1), 13-22$

Madeira, V.M., 1975. A rapid and ultrasensitive method to measure $\mathrm{Ca}^{++}$movements across biological membranes. Biochem. Biophys. Res. Commun. 64 (3), 870 876.

Madeira, V.M., Antunes-Madeira, M.C., Carvalho, A.P., 1974. Activation energies of the ATPase activity of sarcoplasmic reticulum. Biochem. Biophys. Res. Commun. 58 (4), 897-904.

Mandal, P.K., 2005. Dioxin: a review of its environmental effects and its aryl hydrocarbon receptor biology. J. Comp. Physiol. [B] 175 (4), 221-230.

Masini, A., Ceccarelli-Stanzani, D., Muscatello, U., 1984. An investigation on the effect of oligomycin on state-4 respiration in isolated rat-liver mitochondria. Biochim. Biophys. Acta 767 (1), 130-137.

Mbye, L.H., Singh, I.N., Carrico, K.M., Saatman, K.E., Hall, E.D., 2009. Comparative neuroprotective effects of cyclosporin A and NIM811, a nonimmunosuppressive cyclosporin A analog, following traumatic brain injury. J. Cereb. Blood Flow Metab. 29 (1), 87-97.

McGwin, G., Lienert, J., Kennedy, J.I., 2010. Formaldehyde exposure and asthma in children: a systematic review. Environ. Health Perspect. 118 (3), 313-317.

Millay, D.P., Sargent, M.A., Osinska, H., Baines, C.P., Barton, E.R., Vuagniaux, G., Sweeney, H.L., Robbins, J., Molkentin, J.D., 2008. Genetic and pharmacologic inhibition of mitochondrial-dependent necrosis attenuates muscular dystrophy. Nat. Med. 14 (4), 442-447.

Mohammadi-Bardbori, A., Ghazi-Khansari, M., 2008. Alternative electron acceptors: proposed mechanism of paraquat mitochondrial toxicity. Environ. Toxicol. Pharmacol. 26, 1-5.

Nicholls, D.G., 2009. Mitochondrial calcium function and dysfunction in the central nervous system. Biochim. Biophys. Acta 1787 (11), 1416-1424.

Orrenius, S., Gogvadze, V., Zhivotovsky, B., 2007. Mitochondrial oxidative stress: implications for cell death. Annu. Rev. Pharmacol. Toxicol. 47, 143-183.
Palmeira, C.M., Madeira, V.M., 1997. Mercuric chloride toxicity in rat liver mitochondria and isolated hepatocytes. Environ. Toxicol. Pharmacol. 3 (3) 229-235.

Palmeira, C.M., Wallace, K.B., 1997. Benzoquinone inhibits the voltage-dependent induction of the mitochondrial permeability transition caused by redox-cycling naphthoquinones. Toxicol. Appl. Pharmacol. 143 (2), 338-347.

Palmeira, C.M., Moreno, A.J., Madeira, V.M., 1994. Interactions of herbicides 2,4-D and dinoseb with liver mitochondrial bioenergetics. Toxicol. Appl. Pharmacol. 127 (1), 50-57.

Pastorino, J.G., Hoek, J.B., 2008. Regulation of hexokinase binding to VDAC. J. Bioenerg. Biomembr. 40 (3), 171-182.

Raaschou-Nielsen, O., Andersen, Z.J., Hvidberg, M., Jensen, S.S., Ketzel, M., Sørensen, M., Loft, S., Overvad, K., Tjønneland, A., 2011. Lung cancer incidence and longterm exposure to air pollution from traffic. Environ. Health Perspect. 119 (6), 860-865.

Rasola, A., Sciacovelli, M., Pantic, B., Bernardi, P., 2010. Signal transduction to the permeability transition pore. FEBS Lett. 584 (10), 1989-1996.

Rico de Souza, A., Zago, M., Pollock, S.J., Sime, P.J., Phipps, R.P., Baglole, C.J., 2011 Genetic ablation of the aryl hydrocarbon receptor causes cigarette smokeinduced mitochondrial dysfunction and apoptosis. J. Biol. Chem. 286, 4321443228.

Rolo, A.P., Teodoro, J.S., Peralta, C., Rosello-Catafau, J., Palmeira, C.M., 2009 Prevention of $I / R$ injury in fatty livers by ischemic preconditioning is associated with increased mitochondrial tolerance. the key role of ATPsynthase and mitochondrial permeability transition. Transpl. Int. 22 (11), 1081-1090.

Roos, D.H., Puntel, R.L., Farina, M., Aschner, M., Bohrer, D., Roca, J.B., de Vargas Barbosa, N.B., 2011. Modulation of methylmercury uptake by methionine: prevention of mitochondrial dysfunction in rat liver slices by a mimicry mechanism. Toxicol. Appl. Pharmacol. 252 (1), 28-35.

Russmann, S., Kullak-Ublick, G.A., Grattagliano, I., 2009. Current concepts of mechanisms in drug-induced hepatotoxicity. Curr. Med. Chem. 16 (23), 3041 3053.

Sang, H., Zhang, L., Li, J., 2012. Anti-benzopyrene-7,8-diol-9,10-epoxide induces apoptosis via mitochondrial pathway in human bronchiolar epithelium cells independent of the mitochondria permeability transition pore. Food Chem. Toxicol. 50 (7), 2417-2423.

Shertzer, H.G., Genter, M.B., Shen, D., Nebert, D.W., Chen, Y., Dalton, T.P., 2006. TCDD decreases ATP levels and increases reactive oxygen production through changes in mitochondrial $\mathrm{F}(0) \mathrm{F}(1)$-ATP synthase and ubiquinone. Toxicol. Appl. Pharmacol. 217, 363-374.

Sileikytė, J., Petronilli, V., Zulian, A., Dabbeni-Sala, F., Tognon, G., Nikolov, P. Bernardi, P., Ricchelli, F., 2011. Regulation of the inner membrane mitochondrial permeability transition by the outer membrane translocator protein (peripheral benzodiazepine receptor). J. Biol. Chem. 286 (2), 1046-1053.

Silva, F.S.G., Ribeiro, M.P.C., Santos, M.S., Rocha-Pereira, P., Santos-Silva, A., Custódio, J.B.A., 2013. Acitretin affects bioenergetics of liver mitochondria and promotes mitochondrial permeability transition: potential mechanisms of hepatotoxicity. Toxicology 306, 93-100.

Simões, A.M., Duarte, F.V., Teodoro, J.S., Rolo, A.P., Palmeira, C.M., 2010. Exposure to 2,3,7,8-tetrachlorodibenzo- $p$-dioxin and tetraethyl-lead affects lung mitochondria bioenergetics. Toxicol. Mech. Methods 20 (1), 1-6.

Tang, Y., Wang, F., Jin, C., Liang, H., Zhong, X., Yang, Y., 2013. Mitochondrial injury induced by nanosized titaniumdioxide in A549 cells and rats. Environ. Toxicol. Pharmacol.

Teodoro, J.S., Simões, A.M., Duarte, F.V., Rolo, A.P., Murdoch, R.C., Hussain, S.M., Palmeira, C.M., 2011. Assessment of the toxicity of silver nanoparticles in vitro: a mitochondrial perspective. Toxicol. In Vitro 25 (3), 664-670.

Varela, A.T., Gomes, A.P., Simões, A.M., Teodoro, J.S., Duarte, F.V., Rolo, A.P., Palmeira, C.M., 2008. Indirubin-3'-oxime impairs mitochondrial oxidative phosphorylation and prevents mitochondrial permeability transition induction. Toxicol. Appl. Pharmacol. 233 (2), 179-185.

Varela, A.T., Simões, A.M., Teodoro, J.S., Duarte, F.V., Gomes, A.P., Palmeira, C.M., Rolo, A.P., 2010. Indirubin-3'-oxime prevents hepatic I/R damage by inhibiting GSK-3beta and mitochondrial permeability transition. Mitochondrion 10 (5), 456-463.

Yoshioka, W., Peterson, R.E., Tohyama, C., 2011. Molecular targets that link dioxin exposure to toxicity phenotypes. J. Steroid Biochem. Mol. Biol. 127 (1-2), 96101. 